of a larger sample of tetrads will therefore be expected to provide a significant deviation from $1: 2: 1$ if there is chromatid interference. However, from Strickland's published data it is not justifiable to conclude that there is such interference, since hypothesis (1) above has not been ruled out. In other words, the 'correcting' of chromatid interference data to allow for triple cross-overs can be used for estimating the magnitude of such interference but not for detecting its occurrence in the first place.

Botany School,

University of Cambridge. Aug. 11.

1 Strickland, W. N., Proc. Roy. Soc., B, 149, 82 (1958).

- Whitehouse, H. L. K., C.R. Lab. Carlsberg, 26, 407 (1956).

\section{Cytochromes of Rhizobium}

LEGHAMOGLOBIN, the red pigment associated with the bacteroid form of Rhizobium in effective legume root nodules, appears to be closely linked with the nitrogen fixation process ${ }^{1}$, but its connexion with Rhizobium respiration is disputed ${ }^{2,3}$. We are endeavouring to clarify the latter problem by a comparative study of respiratory pigments in several free-living Rhizobium strains and the related root nodules.

While observing with a microspectroscope the reduction of leghæmoglobin in fresh homogenates of effective soybean or clover nodules made anaerobic by endogenous respiration, we saw also a sharp absorption band appearing near $551 \mathrm{~m} \mu$. This was later identified as the $\alpha$-absorption band of reduced bacteroid cytochrome $c$, and it therefore appeared that biological reduction of the two pigments could be closely related. A smaller amount of b-type cytochrome, with $\alpha$-absorption band at $562 \mathrm{~m} \mu$, was also seen in the washed bacteroids isolated from these homogenates, but no cytochrome $a$ could be detected even when dense bacteroid suspensions in 50 per cent $(\mathrm{v} / \mathrm{v})$ glycerol were examined in the spectroscope at liquid air temperature (ef. Hartree ${ }^{4}$ ). Typical cytochrome $a, b$ and $c$ absorption bands as described by Smith $^{5}$ were readily observed in the same Rhizobia grown on an agar medium, showing that, in the transition from free-living to bacteroid form, Rhizobium loses its cytochrome $a$.

Many micro-organisms suffer an adaptive loss of cytochrome $a$ when grown anaerobically ${ }^{6}$, and as the nodule interior is assumed to be at a very low oxygen tension ${ }^{2}$ we then made a quantitative comparison of cytochrome components in effective soybean nodule bacteria and the same Rhizobium strain grown in pure culture at high and low oxygen tensions. Cytochrome concentrations were measured in a Bellingham and Stanley reversion microspectroscope following the general procedure described by Hartre $e^{4}$. The comparison prism of this instrument was remounted so that the bacterial spectrum and the comparison spectrum (of reduced mammalian cytochrome $c$ contained in a wedge-trough) were viewed simultaneously. By turning the reversion screw, the $\alpha$-absorption band of the standard cytochrome $c$ was matched in turn against each cytochrome band of the bacterial spectrum. An arbitrary assumption was made that the $\alpha$-absorption bands of all components had the same specific extinction coefficient as cytochrome $c$.
These measurements (Table 1, A) showed an increase of cytochromes $b$ and $c$ in effective nodule bacteria (bacteroids) and cultured Rhizobium grown at low oxygen tension, and confirmed the complete loss of cytochrome $a$ from bacteroids. Cytochrome $a$ had decreased in amount, but not disappeared from the cultured organisms grown at an oxygen pressure of $0.02 \mathrm{~atm}$. We were unable to grow Rhizobium in liquid culture at oxygen tensions lower than this and so could not decide whether the complete disappearance of cytochrome $a$ from bacteroids is solely due to a very low oxygen tension inside the effective nodule. Subsequent measurements (Table 1,B) on Rhizobium isolated from ineffective soybean nodules (which were devoid of leghæmoglobin and caused no detectable nitrogen fixation), made this explanation seem unlikely, as these bacteria still contained a small amount of cytochrome $a$.

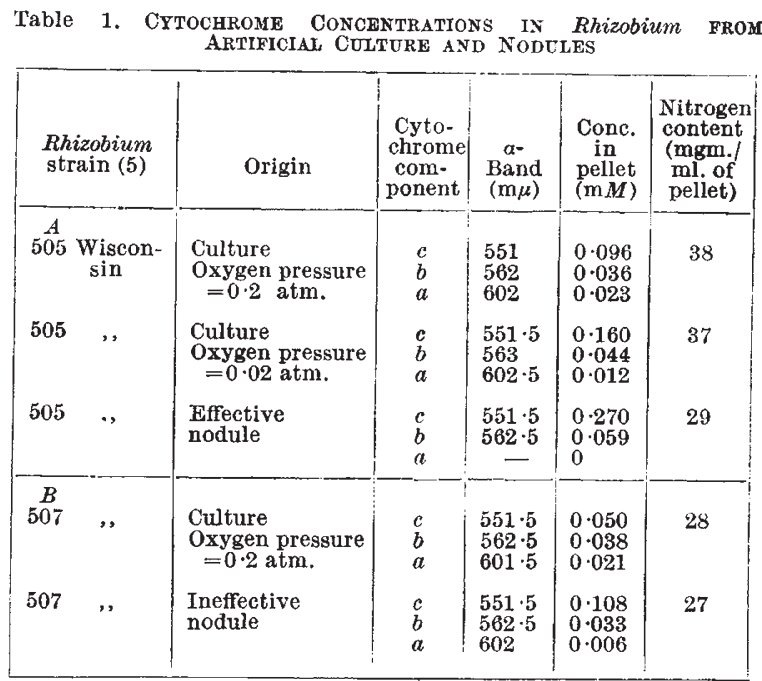

The bacteria were isolated from vigorously aerated log-phase liquid cultures and from 6-7-week-old nodules of 'Lincoln' strain soybean grown in the glasshouse. After checking for purity the washed Rhizobium suspenslons were reduced with dithionite, and the pellets obtained after high-speed centrifuging $(30,000 g)$ were placed in demountable spectrophotometer cuvettes of 1,2 or $3 \mathrm{~mm}$. light path. Cytochrome contents, determined as described in the text, are expressed as millimolar concentrations in the packed pellets. Total nitrogen
values were determined by the Kjeldahl method.

Work is at present in progress to determine what function cytochrome $a$ has in the terminal oxidase system of Rhizobium, and whether its unique disappearance from bacteroids is accompanied by the appearance of another oxidase which might react specifically with legh: moglobin.

\section{A. Appleby}

F. J. Bergersen

Division of Plant Industry,

Commonwealth Scientific and

Industrial Research Organization,

Canberra.

Aug. 13.

'Virtanen, A. I., Erkama, J., and Linkola, H., Acta Chem. Scand., 1 $861(1947)$.

${ }^{2}$ Smith, J. D., Biochem. J., 44, 591 (1949).

s Burris, R. H., and Wilson, P. W., Biochem. J., 51, 90 (1952).

- Hartree, E. F., in "Modern Methods of Plant Analysis", vol. 4, p. 197. Ed.' by Paech, K., and Tracey, M. V. (Springer-Verlag, Berlin, 1955).

${ }^{5}$ Smith, J. D., Biochem. J., 44, 585 (1949).

${ }^{6}$ Chaix, P., and Heyman-Blanchet, T., Biochim. et Biophys. Acta, 28, $214(1957)$.

'Smith, L., Bact. Rev., 18, 106 (1954). 